HYPOTHESIS

\title{
DFNA5: hearing impairment exon instead of hearing impairment gene?
}

\author{
L Van Laer, K Vrijens, S Thys, V F I Van Tendeloo, R J H Smith, D R Van Bockstaele, \\ J-P Timmermans, G Van Camp
}

See end of article for authors' affiliations

....................

Correspondence to:

G Van Camp, Department

of Medical Genetics,

University of Antwerp,

Campus Drie Eiken,

Universiteitsplein 1,

B-2610 Antwerp, Belgium;

Guy.VanCamp@ua.ac.be

Revised version received 30 October 2003

Accepted for publication

2 November 2003

\begin{abstract}
Background: Three mutations in the DFNA5 gene have been described in three families with autosomal dominant non-syndromic hearing impairment. Although these mutations are different at the genomic DNA level, they all lead to skipping of exon 8 at the mRNA level. We hypothesise that hearing impairment associated with DFNA5 is caused by a highly unusual mechanism, in which skipping of one specific exon leads to disease that is not caused by other mutations in this gene. We hypothesise that this represents a very specific "gain of function" mutation, with the truncated protein exerting a deleterious new function. Methods: We performed transfection experiments in mammalian cell lines (HEK293T and COS-1) with green fluorescent protein (GFP) tagged wildtype and mutant DFNA5 and analysed cell death with flow cytometry and fluorescence microscopy.

Results: Post-transfection death of HEK293T cells approximately doubled when cells were transfected with mutant DFNA5-GFP compared with wildtype DFNA5-GFP. Cell death was attributed to necrotic events and not to apoptotic events.

Conclusion: The transfection experiments in mammalian cell lines support our hypothesis that the hearing impairment associated with DFNA5 is caused by a "gain of function" mutation and that mutant DFNA5
\end{abstract} has a deleterious new function.
$\mathrm{D}$ FNA5 was first identified in an extended Dutch family that long remained the only family in which a mutation in DFNA5 was discovered. ${ }^{1}$ This changed very recently, with the description of a Chinese family and a second Dutch family with mutations in the DFNA5 gene. ${ }^{2}$ The hearing loss in the three families is similar: progressive, non-syndromic, sensorineural, and starting in the high frequencies. The age of onset differs slightly, ranging from 0 to 40 years in the newly described Dutch family, over 5 to 15 years in the original Dutch family, and from 7 to 30 years in the Chinese family. ${ }^{1-3}$ The mutations in DFNA5 that lead to this hearing loss are diverse: a complex insertion-deletion mutation in intron 7, a deletion of three nucleotides in the polypyrimidine tract of intron 7 , and a nucleotide substitution in the splice acceptor site of intron 7 (fig 1). ${ }^{1-3}$ Most interestingly, however, at the mRNA level, all of these mutations lead to skipping of exon 8, which indicates that this particular event only might cause hearing impairment. It cannot be a coincidence that, despite being different at the level of DNA, all three currently known mutations of DFNA5 lead to skipping of exon 8. Mutations in other parts of DFNA5 might have no clinical effect at all or might result in a completely different phenotype. The fact that skipping of one specific exon in a gene leads to a particular monogenic phenotype that is not caused by other mutations in this gene is a highly unusual finding and, to the best of our knowledge, is unprecedented.

Previously, we had assumed that hearing impairment in the Dutch family was due to haploinsufficiency. This assumption resulted from studies in a mouse model that was generated by the deletion of exon 8 by targeted recombination in embryonic stem cells to mimic the human mutation. The presence of aberrant mRNA was shown by reverse transcriptase polymerase chain reaction (PCR) in this mouse model. No protein could be detected in homozygous DFNA5 $^{-/}$mice with Western blotting, however, which indicates that the aberrant mRNA probably was destroyed through nonsense mediated decay or, alternatively, that the aberrant protein was unstable (unpublished data). It was tempting to speculate that an identical process occurs in affected family members; however, the observation that most probably only skipping of exon 8 leads to hearing impairment is not in line with the haploinsufficiency hypothesis and points towards a "gain of function" mutation.

In contrast with mouse aberrant mRNA, human aberrant mRNA might escape detection by the nonsense mediated decay machinery. A possible reason for this escape might be the presence of the premature termination codon in the final part of the protein; DFNA5 contains only 10 exons. The reason for this difference in detection between humans and mice is unclear. Human aberrant mRNA might get translated, which introduces an aberrant stretch of 41 amino acids after amino acid 330, which could lead to a new protein product that might display a different function from its wildtype counterpart. A first line of evidence for a deleterious new function of human mutant DFNA5 was gained very recently with a yeast model for the study of human DFNA5. Gregan et al showed that the expression of human mutant DFNA5 in yeast cells caused loss of viability of the yeast cells, while expression of human wildtype DFNA5 did not harm the yeast cells. ${ }^{4}$ Further proof for our hypothesis was gathered during the transfection experiments described below.

\section{MATERIAL AND METHODS}

\section{Cell cultures}

We grew HEK293T cells and COS-1 cells in Dulbecco's modified Eagle's medium with $4500 \mathrm{ml} / \mathrm{l}$ glucose (Invitrogen, San Diego, CA, USA) supplemented with 15\% (volume/ volume) fetal calf serum (Invitrogen, San Diego, CA, USA), $100 \mathrm{U} / \mathrm{ml}$ penicillin, $100 \mu \mathrm{g} / \mathrm{ml}$ streptomycin (Invitrogen, San Diego, CA, USA), and $2 \mathrm{mM}$ L glutamine (Invitrogen, San Diego, CA, USA) in a humidified atmosphere of $5 \%$ carbon dioxide at $37^{\circ} \mathrm{C}$. 


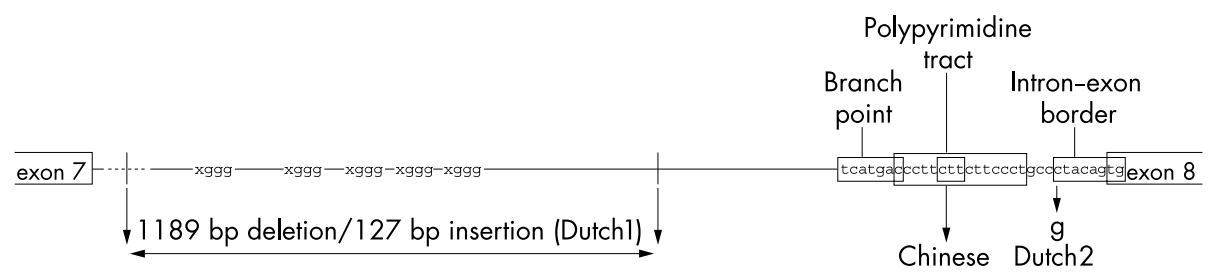

Figure 1 Representation of the three different DFNA5 mutations in intron 7. The original Dutch mutation (Dutch 1) deletes a 1189 bp fragment and inserts a 127 bp fragment from intron 8 in the opposite direction followed by a GCCCA stretch from unknown origin. The assumed cause of exon 8 skipping is the deletion of five intronic G triplets, represented by XGGG.' The Chinese mutation deletes one of three CTT repeats in the polypyrimidine tract. ${ }^{2}$ Finally, the new Dutch mutation (Dutch 2) is a C>G substitution in the intron-exon border consensus sequence. ${ }^{3}$ The putative "adenine for lariat formation" in the branch point consensus sequence is indicated with an asterisk.

Generation of human wildtype and mutant DFNA5green fluorescent protein (GFP) constructs

We cloned mRNA of human wildtype and mutant DFNA5 by PCR into pEGFP-Nl (Clontech, BD Biosciences, Palo Alto, CA, USA) with the Advantage cDNA PCR kit (Clontech, BD Biosciences, Palo Alto, CA, USA) and the primers 5'-CGGGCCCGGGATCCAATGTTTGCCAAAGCAACCAGG-3' and 5'-CGGTGGATCCCGTGAATGTTCTCTGCCTAAAGC-3' for wildtype DFNA5 and 5'-AAGCTTCGAATTCTGATGTTTG CCAAAGCAACCAGG-3' and 5'-CGGTGGATCCCGAGGTT GGGTCTTCAAGATCAG-3' for mutant DFNA5. The PCR reaction consisted of 5 minutes of denaturation at $94^{\circ} \mathrm{C}$, 30 cycles of 1 minute denaturation at $94^{\circ} \mathrm{C}, 1$ minute of annealing at $55^{\circ} \mathrm{C}$, a 2 minute extension at $72^{\circ} \mathrm{C}$ for wildtype DFNA5 or a 1 minute extension at $72^{\circ} \mathrm{C}$ for mutant DFNA5, and a 10 minute extension at $72^{\circ} \mathrm{C}$. Templates for the PCR reactions were the previously described human full length and mutant DFNA5 clone, respectively. ${ }^{4}$ Before ligation, PCR fragments and pEGFP-Nl vector were digested with BamHI or double digested with BamHI and EcoRI for wildtype DFNA5 and mutant DFNA5, respectively. We sequenced inserts completely bidirectionally to exclude the introduction of cloning errors.

\section{Transfection experiments}

For confocal microscopy, we cultivated both cell lines on sterile cover slides (12 mm diameter; $0.13-0.17 \mathrm{~mm}$ ) in $60 \mathrm{~mm}$ tissue culture dishes with about $2 \times 10^{5}$ cells per dish at $37^{\circ} \mathrm{C}$ and $5 \%$ carbon dioxide for $18-24$ hours. We incubated transfection solutions that contained no DNA (for mock transfected cells), $500 \mathrm{ng}$ DNA (for transfections with wildtype DFNA5-GFP, mutant DFNA5-GFP, or empty vector indicated with GFP) for HEK293T cells, or $1 \mu \mathrm{g}$ DNA for COSl cells; $12 \mu \mathrm{l}$ Lipofectamine reagent (Invitrogen, San Diego, CA, USA); and $500 \mu \mathrm{l}$ Optimem 1 (Invitrogen, San Diego, CA, USA) for 30 minutes at $37^{\circ} \mathrm{C}$. We rinsed the cover slides twice in Optimem 1. After we added another $2 \mathrm{ml}$ of Optimem 1 to the transfection solution, we incubated the cells in the transfection solution for 6 hours at $37^{\circ} \mathrm{C}$ in $5 \%$ carbon dioxide. Subsequently, we incubated the cover slides in normal growth medium for 18-24 hours. We visualised GFP fluorescent cells with a Zeiss CLSM 510 confocal laser scanning microscope (Zeiss, Göttingen, Germany) equipped with an argon laser (excitation, $488 \mathrm{~nm}$ ).

For flow cytometry, we grew and transfected cells directly in tissue culture dishes under similar conditions as described above. After 18-24 hours of incubation, we harvested culture medium containing floating cells. We harvested adherent cells by trypsinisation (trypsin-ethylene diamine tetraacetic acid, Invitrogen, San Diego, CA, USA), pooled them with the floating cells, and centrifuged them for 10 minutes at $1400 \mathrm{rpm}$ to remove culture medium. We resuspended the cell pellet in $500 \mu \mathrm{l}$ of sterile phosphate buffered saline and $0.1 \%$ bovine serum albumin. Before flow cytometric analysis, we added $1 \mu \mathrm{g} / \mu \mathrm{l}$ ethidium bromide to the cells and analysed
GFP expression and viability (measured by exclusion of ethidium bromide) on a FACScan analytical flow cytometer (BD Biosciences, San Jose, CA, USA) with CELLQuest software (BD Biosciences, San Jose, CA, USA).

\section{Double staining with Hoechst 33342 and ethidium bromide}

Twenty-four hours after transfection, we incubated cells with $5 \mu \mathrm{g} / \mathrm{ml}$ of Hoechst 33342 and ethidium bromide. We incubated cells for 15 minutes at $37^{\circ} \mathrm{C}$ in $5 \%$ carbon dioxide. We then scored cells with a Fluovert fluorescence microscope according to the following criteria: viable cells displayed a faint blue (Hoechst 33342) fluorescent staining, apoptotic cells an intense blue staining in combination with the presence of apoptotic bodies, necrotic cells a red (ethidium bromide) staining, and secondary necrotic cells (or late apoptotic cells) a red staining in the presence of apoptotic bodies. $^{5}$

\section{Statistical analysis}

We tested differences between transfections with wildtype or mutant DFNA5 with $\chi^{2}$ statistics if at least $80 \%$ of the expected values exceeded five and all the expected values exceeded one. If the expected values did not meet these criteria, we added the least frequent values and applied $\chi^{2}$ statistics to the resulting figures. In case the addition of infrequent values resulted in a two by two table, we used Yates's correction for continuity. We calculated statistics with SigmaStat software (version 2; Jandel Corporation, San Rafel, CA, USA).

\section{RESULTS AND DISCUSSION}

Further evidence for our hypothesis that human mutant DFNA5 might exert a deleterious new function was gathered during experiments originally initiated to elucidate the subcellular localisation of wildtype and mutant DFNA5. PSORTII predicted the most probable subcellular localisation for DFNA5 to be cytoplasmic, ${ }^{6}$ while prediction of transmembrane regions with PSORTII and Tmpred (http:// www.ch.embnet.org/software/TMPRED) resulted in a varying number of transmembrane regions with relatively low scores. Although the most probable subcellular localisation for mutant DFNA5 remained cytoplasmic, a high scoring transmembrane region was predicted in the aberrant tail of the mutant DFNA5 protein.

To test these predictions experimentally, we tagged wildtype and mutant DFNA5 with GFP and transfected constructs into COS-1 and HEK293T cell lines. Wildtype DFNA5-GFP was distributed smoothly in the cytoplasm, while mutant DFNA5-GFP was localised in the cytoplasm in a granular manner in HEK293T cells (fig 2A) and in COS-1 cells (data not shown). In addition to its cytoplasmic localisation, mutant DFNA5-GFP seemed to be associated with the plasma membrane in HEK293T cells, especially in cells with an 


\section{A}
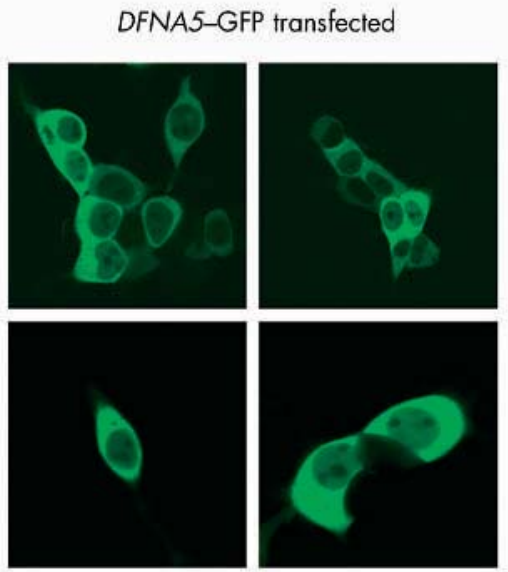

\section{Mutant DFNA5-GFP transfected}
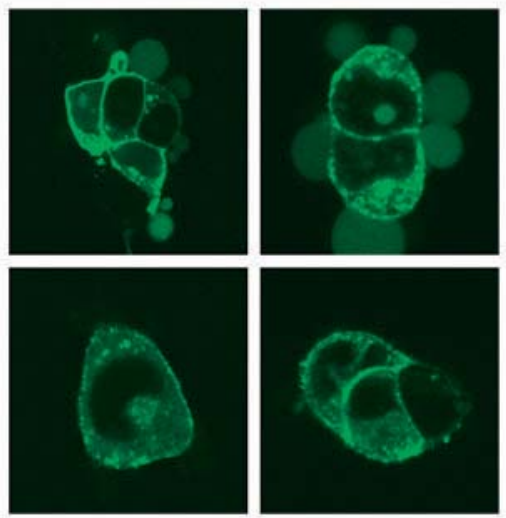

B

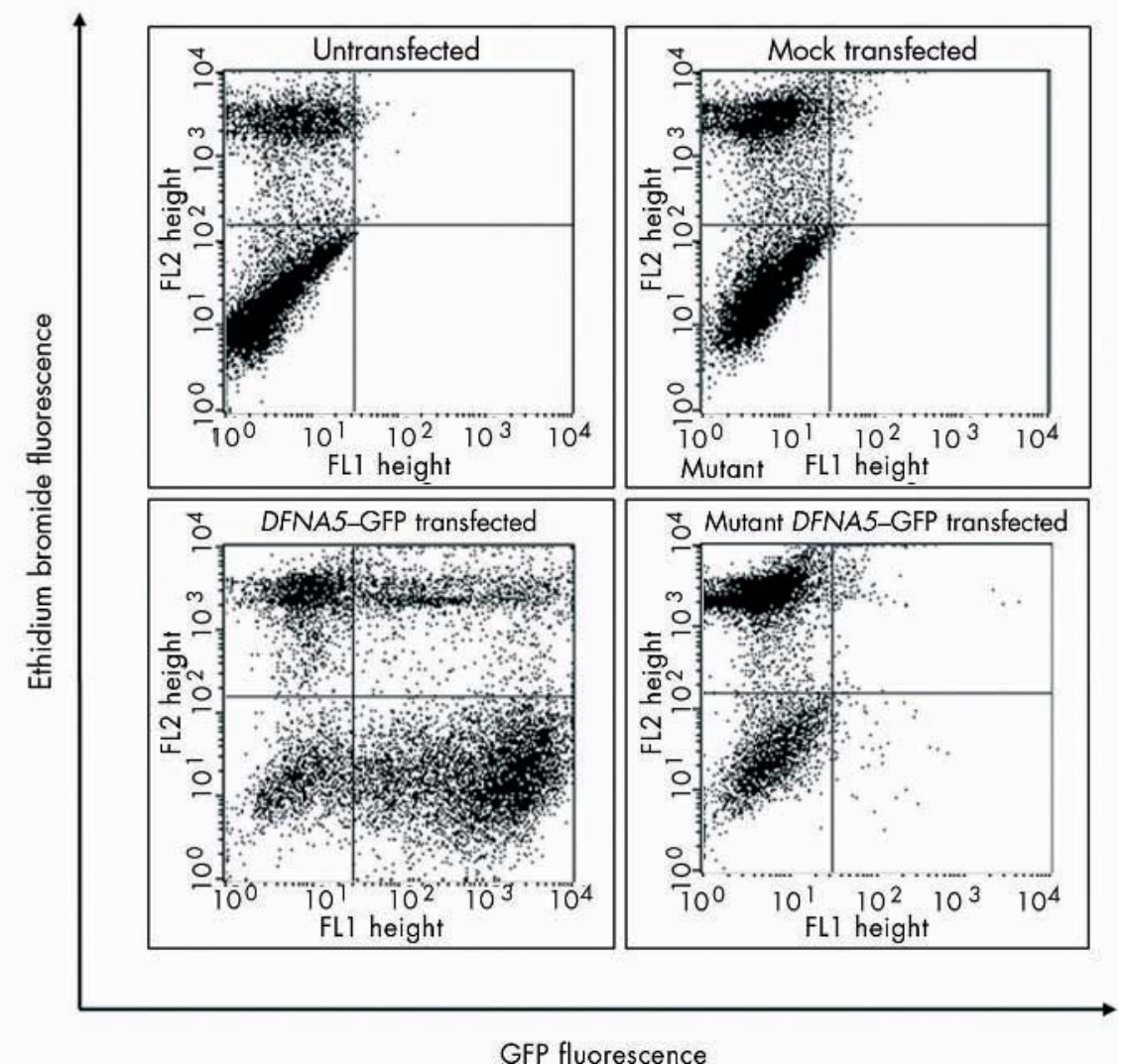

Figure 2 Transfection experiments in HEK293T cells. Twenty-four hours after transfection, we (A) subjected HEK293T cells to fluorescence and confocal microscopy or (B) trypsinised them and studied them in the presence of ethidium bromide with FACScan flow cytometry. In the dot plots, the lower left quadrant represents viable GFP' cells, the lower right viable GFP' cells, the upper left dead GFP' cells, and the upper right dead GFP' cells. Similar results were obtained with COS-1 cells (data not shown). Table 1 gives the percentages of different cell counts on the basis of the number of dots in the quadrant analysis.

unhealthy appearance (a more circular shape rather than a stretched shape and with cell blebbing) (fig 2A). This phenomenon was not seen in COS-1 cells (data not shown). The plasma membrane associated localisation of mutant DFNA5-GFP in HEK293T cells might indicate that the predicted high scoring transmembrane region in the aberrant tail is functional or that mutant DFNA5 associates with a membrane protein. The latter might be present in HEK293T cells but absent in COS-1 cells. Alternatively, membrane associated localisation of mutant DFNA5-GFP might only be detected in the presence of high concentrations of mutant protein that might never be reached with the current transfection conditions for COS-1 cells. Overall, transfections with mutant DFNA5-GFP seemed less efficient than those with wildtype DFNA5-GFP, and fluorescence always was weaker.

In view of the results obtained in yeast cells and the fluorescence microscopy results described above, ${ }^{4}$ we assumed that our observations might be the result of the cell death of mutant DFNA5-GFP transfected cells. This hypothesis was confirmed with flow cytometry (fig 2B and table 1). Even when we took into account cell death because 
Table 1 Cell counts on the basis of the number of dots in quadrant analysis for HEK293T and COS-1 cells*

\begin{tabular}{|c|c|c|c|c|c|c|}
\hline \multirow[b]{2}{*}{ Cells } & \multicolumn{3}{|c|}{ Viable (\%) } & \multicolumn{3}{|c|}{ Dead $(\%)$} \\
\hline & GFP $^{-}$ & $\mathrm{GFP}^{+}$ & Total & GFP $^{-}$ & $\mathrm{GFP}^{+}$ & Total \\
\hline \multicolumn{7}{|l|}{ HEK293T } \\
\hline Untransfected cells & 87.88 & 0.00 & 87.88 & 12.10 & 0.02 & 12.12 \\
\hline Mock transfected cells & 72.47 & 0.13 & 72.60 & 26.30 & 1.10 & 27.40 \\
\hline 500 ng DFNA5-GFP & 15.09 & 53.71 & 68.80 & 20.26 & 10.94 & 31.20 \\
\hline 500 ng mutant DFNA5-GFP & 16.92 & 6.87 & 23.79 & 32.59 & 43.62 & 76.21 \\
\hline \multicolumn{7}{|l|}{$\cos -1$} \\
\hline Untransfected cells & 95.09 & 0.01 & 95.10 & 4.87 & 0.03 & 4.90 \\
\hline Mock transfected cells & 92.55 & 0.09 & 92.64 & 7.17 & 0.19 & 7.36 \\
\hline $1 \mu \mathrm{g}$ DFNA5-GFP & 79.16 & 11.27 & 90.43 & 8.63 & 0.94 & 9.57 \\
\hline $1 \mu \mathrm{g}$ mutant DFNA5-GFP & 84.74 & 4.56 & 89.30 & 9.11 & 1.59 & 10.70 \\
\hline \multicolumn{7}{|c|}{$\begin{array}{l}\text { *In total, } 10^{4} \text { cells were analysed with CELLQuest software. Numbers are deduced from one representative experiment out of two experiments (dot plots are shown } \\
\text { in fig } 2 \text { B). With } \chi^{2} \text { statistics, p values of }<0.001 \text { and } 0.171 \text { were calculated in HEK293T and COS- } 1 \text { cells, respectively, for the differences between transfections } \\
\text { with wildtype DFNA5-GFP and mutant DFNA5-GFP for the experiment represented in table } 1 \text { and fig } 2 \text {. The second experiment gave } p \text { values of }<0.001 \text { and } \\
0.027 \text {, respectively. }\end{array}$} \\
\hline
\end{tabular}

of trypsinisation (12\% in HEK293T cells and 5\% in COS-1 cells) and lipofectamine (another 15\% in HEK293T cells and $2 \%$ in COS- 1 cells, the percentage of dead HEK293T cells was significantly higher in cells transfected with mutant DFNA5GFP than in cells transfected with wildtype DFNA5-GFP (table 1). In COS-1 cells, the effect was less obvious. This might be because of a reduced transfection efficiency (12\% compared with 65\% in HEK293T cells, as deduced from wildtype DFNA5-GFP transfections), which was even lower when mutant DFNA5-GFP was used (6\%) (table 1). In general, COS- 1 cells seemed to be more resistant to stress factors, including trypsinisation and lipofectamine, and more reluctant to uptake mutant DFNA5-GFP. The ratio of $\mathrm{GFP}^{+}$ dead cells to $\mathrm{GFP}^{+}$viable cells in mutant DFNA5-GFP transfected COS-1 cells (0.35) was still significantly higher, however, when compared with that for COS-1 cells transfected with wildtype DFNA5-GFP (0.08). Cell death attributed to mutant DFNA5-GFP thus was seen in both cell lines.

As DFNA5 has been implicated in signalling pathways that lead to apoptosis, ${ }^{7}$ we investigated whether the observed cell death was the consequence of apoptotic or necrotic events with a double staining procedure with Hoechst 33342 and ethidium bromide. Viable cells displayed a faint blue (Hoechst 33342) fluorescent staining, apoptotic cells an intense blue staining in combination with the presence of apoptotic bodies, necrotic cells a red (ethidium bromide) staining, and secondary necrotic cells (or late apoptotic cells) a red staining in the presence of apoptotic bodies (fig 3 and table 2). Table 2 clearly shows that the number of necrotic cells was significantly higher in cells transfected with mutant
DFNA5-GFP, while the number of apoptotic cells remained stable. Again, the results were less significant in COS-1 cells.

Protein overexpression after transfection of cells hardly is comparable to physiological circumstances. Massive cell death thus is unlikely to occur in vivo in carriers of DFNA5 mutations. At low physiological levels of expression, mutant DFNA5 is more likely to display a toxic effect on cells without killing them immediately, instead rendering them more susceptible to certain stress factors or ageing. Hearing loss associated with DFNAS could be caused by the same pathological mechanisms as age related hearing loss. Indeed, phenotypically, they are alike: both start at the high frequencies and are progressive, sensorineural, and bilateral. The main difference is the age at onset. A model that possibly resembles the in vivo situation more closely might be that of a transgenic mouse that expresses human mutant DFNA5 at physiological levels. Such a model might be a good way to study pathological mechanisms that lead to hearing loss.

Expression of DFNA5 was detected at low levels in every tissue investigated so far (reference 1 and unpublished results). If cell death occurred in every tissue that expressed $D F N A 5$, a lethal phenotype would be expected in mutation carriers. Affected people show progressive hearing impairment as the only recognisable clinical characteristic. Cochlear cells may be much more sensitive to the deleterious actions of mutant DFNA5 because the latter might interact with cellular components that are expressed only in cochlear cells. Whether these cellular components also are involved in normal DFNA5 activity or part of completely different pathways remains to be elucidated.
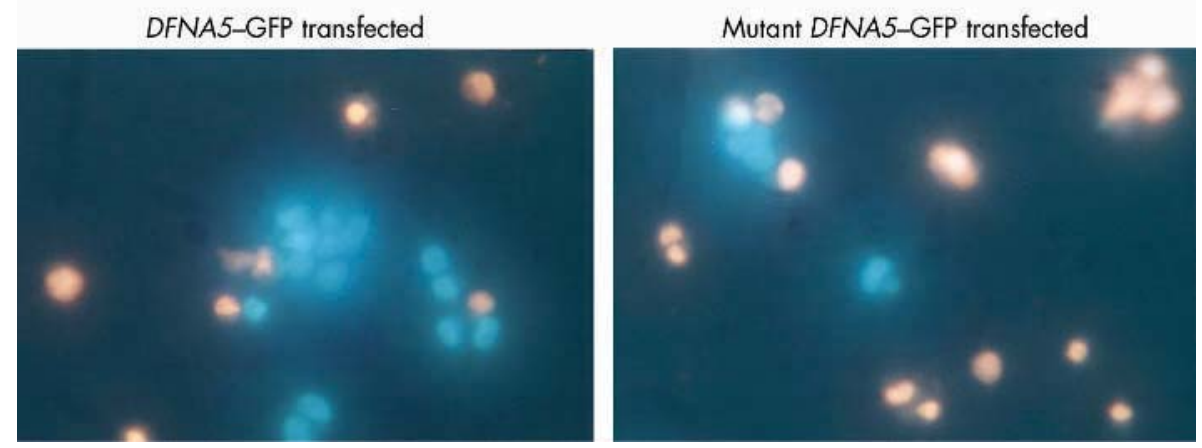

Figure 3 Hoechst 33342 and ethidium bromide double staining of transfected HEK293T cells. Twenty-four hours after transfection, cells were double stained with Hoechst 33342 and ethidium bromide, after which they were scored by fluorescence microscopy. Representative pictures were chosen for this figure, so only viable (blue) cells and necrotic (red) cells can be seen. No apoptotic or late apoptotic cells are present in the represented fields. Similar results were obtained with COS-1 cells (data not shown). Table 2 gives the percentages of the different cell counts. 
Table 2 Hoechst 33342 and ethidium bromide double staining of transfected HEK293T and COS-1 cells*

\begin{tabular}{|c|c|c|c|c|}
\hline Cells & Viable (\%) & Apoptotic (\%) & Late apoptotic (\%) & Necrotic (\%) \\
\hline \multicolumn{5}{|l|}{ HEK293T } \\
\hline Untransfected cells & 98.44 & 0.78 & 0.33 & 0.45 \\
\hline Mock transfected cells & 86.96 & 0.69 & 0.23 & 12.13 \\
\hline 500 ng GFP & 79.22 & 0.65 & 0.86 & 19.28 \\
\hline 500 ng DFNA5-GFP† & 79.10 & 0.45 & 0.67 & 19.78 \\
\hline 500 ng mutant DFNA5-GFP† & 38.40 & 1.40 & 0.25 & 59.96 \\
\hline \multicolumn{5}{|l|}{$\cos -1$} \\
\hline Untransfected cells & 99.69 & 0.18 & 0.14 & 0.00 \\
\hline Mock transfected cells & 96.90 & 0.84 & 0.18 & 2.09 \\
\hline $1 \mu \mathrm{g}$ GFP & 97.03 & 1.31 & 0.21 & 1.45 \\
\hline $1 \mu \mathrm{g}$ DFNA5-GFP $†$ & 98.04 & 0.54 & 0.00 & 1.43 \\
\hline $1 \mu \mathrm{g}$ mutant DFNA5-GFP† & 93.71 & 0.88 & 0.22 & 5.19 \\
\hline
\end{tabular}

Toxic protein alterations are not uncommon. They are implicated in the pathology of various neurodegenerative diseases, such as Alzheimer's disease ( $\beta$-amyloid precursor protein), Parkinson's disease ( $\alpha$-synuclein), motor neurone disease (copper-zinc superoxide dismutase), and retinitis pigmentosa (rhodopsin). ${ }^{8-11}$ The polyglutamine expansion disorders, including Huntington's disease, several forms of spinocerebellar ataxia, and dentatorubral and pallidoluysian atrophy, comprise a particular group of neurodegenerative disorders that also are thought to be caused by toxic gain of function mechanisms. ${ }^{12-14}$ Besides Alzheimer disease, various other types of amyloidosis (involving various protein deposits including fibrinogen, apolipoprotein A-1, prion protein, transthyretin, gelsolin, cystatin $\mathrm{C}$, and lysozyme) also are caused by toxic effects of mutated proteins. ${ }^{15} 16$ The toxic effect in amyloidosis and Parkinson's disease seems to be accomplished by the conversion of wildtype protein into toxic precursors or waste products, or both, that accumulate and aggregate to form inclusions that slowly poison cells and tissues. As we saw a granular appearance of mutant DFNA5GFP localised in transfected cells, aggregation of mutant protein might also be part of the pathological mechanisms of mutant DFNA5.

Direct cell death seems to be rare, but few examples exist. In a Caenorhabditis elegans model of late onset neurodegeneration, dominant mutations in mec-4 (mechanosensory abnormal) induced vacuolar swelling and lysis of specific neurons, which caused degeneration of the touch receptor. ${ }^{17}$ Truncations of athrophin-1, the protein involved in dentatorubral and pallidoluysian atrophy, altered the ability of the protein to shuttle between the nucleus and the cytoplasm, which led to abnormal nuclear localisation and subsequent cell toxicity. ${ }^{12}$ In addition, numerous other examples of reported mislocalisations of certain proteins as part of the pathological mechanisms can be found in the literature. We observed an abnormal localisation to the plasma membrane in HEK293T cells transfected with mutant DFNA5. This mislocalisation could form an important part of the pathogenesis of mutant DFNA5. On the other hand, abnormal localisation of mutant DFNA5 to the plasma membrane might be only a secondary effect that is part of the necrotic process caused by the toxic action of mutant DFNA5, during which aggregated proteins might be directed inappropriately towards the plasma membrane just before the cell dies. At this moment, to distinguish between cause and effect is impossible.

\section{Conclusion}

Several lines of evidence suggest that mutant DFNA5 exerts a deleterious new function through a unique mechanism of skipping a specific exon. Transfection experiments in mammalian cell lines provided additional support for this hypothesis, as cell death after transfection approximately doubled in HEK293T cells. Cell death was attributed to necrotic events and not to apoptotic events. Further experiments are needed to elucidate the exact pathological mechanisms that lead to cell death in cells transfected with mutant DFNA5. These experiments might shed light on the pathology that leads to hearing loss in families with DFNA5 mutations.

\section{ACKNOWLEDGEMENTS}

We thank Marc Lenjou for excellent technical assistance in flow cytometry analysis and Erik Fransen for statistical advice. LVL and VFIVT are postdoctoral fellows of the Flemish FWO (Fonds voor Wetenschappelijk Onderzoek).

\section{Authors' affiliations}

L Van Laer, K Vrijens, S Thys, G Van Camp, Department of Medical Genetics, University of Antwerp, Antwerp, Belgium

V F I Van Tendeloo, D R Van Bockstaele, Laboratory of Experimental Haematology, Faculty of Medicine, University of Antwerp, Antwerp University Hospital, Antwerp, Belgium

R J H Smith, Molecular Otolaryngology Research Laboratories, University of lowa, lowa City, lowa

J-P Timmermans, Laboratory of Cell Biology and Histology, University of Antwerp, Antwerp, Belgium

Conflicts of interest: none declared.

Funding: This research was supported in part by a grant of the GSKE (Geneeskundige Stichting Koningin Elisabeth) to GVC and LVL and by a NOI-BOF grant of the University of Antwerp to LVL. This research was performed in the framework of the Interuniversity Attraction Poles programme P5/19 of the Federal Office for Scientific, Technical, and Cultural Affairs, Belgium.

\section{REFERENCES}

1 Van Laer L, Huizing EH, Verstreken M, van Zuijlen D, Wauters JG, Bossuyt PJ, Van de Heyning P, McGuirt WT, Smith RJH, Willems PJ, Legan PK, Richardson GP, Van Camp G. Nonsyndromic hearing impairment is associated with a mutation in DFNA5. Nat Genet 1998;20:194-7.

2 Yu C, Meng X, Zhang S, Zhao G, Hu L, Kong X. A 3-nucleotide deletion in the polypyrimidine tract of intron 7 of the DFNA5 gene causes nonsyndromic hearing impairment in a Chinese family. Genomics 2003;82:575-9.

3 Bischoff AMLC, Luijendijk MWJ, Huygen PLM, van Duijnhoven G, De Leenheer EMR, Oudesluijs G, Van Laer L, Cremers FPM, Cremers CWRJ, Kremer $\mathrm{H}$. A second mutation identified in the DFNA5 gene in a Dutch family. A clinical and genetic evaluation. Audiol Neuro-Otol 2004;9:34-46.

4 Gregan J, Van Laer L, Lieto LD, Van Camp G, Kearsey SE. A yeast model for the study of human DFNA5, a gene mutated in nonsyndromic hearing impairment. Biochim Biophys Acta 2003;1638:179-86.

5 Vermeulen K, Strnad M, Krystof V, Havlicek L, Van der Aa A, Lenjou M, Nijs G, Rodrigus I, Stockman B, van Onckelen H, Van Bockstaele DR, Berneman ZN. Antiproliferative effect of plant cytokinin analogues with an inhibitory activity on cyclin-dependent kinases. Leukemia 2002;16:299-305. 
6 Nakai K, Kanehisa M. A knowledge base for predicting protein localization sites in eukaryotic cells. Genomics 1992;14:897-911.

7 Lage H, Helmbach H, Grottke C, Dietel M, Schadendorf D. DFNA5 (ICERE-1) contributes to acquired etoposide resistance in melanoma cells. FEBS Lett 2001;494:54-9.

8 Citron M, Oltersdorf T, Haass C, McConlogue L, Hung AY, Seubert P, Vigo-Pelfrey C, Lieberburg I, Selkoe DJ. Mutation of the beta-amyloid precursor protein in familial Alzheimer's disease increases beta-protein production. Nature 1992;360:672-4.

9 Hashimoto $M$, Rockenstein E, Masliah E. Transgenic models of alphasynuclein pathology: past, present, and future. Ann N Y Acad Sci 2003;991:171-88.

10 Kirby J, Menzies FM, Cookson MR, Bushby K, Shaw PJ. Differential gene expression in a cell culture model of SOD1-related familial motor neurone disease. Hum Mol Genet 2002;1 1:2061-75.

11 Mclnnes RR, Bascom RA. Retinal genetics: a nullifying effect for rhodopsin. Nat Genet 1992;1:155-7.
12 Nucifora FC, Ellerby LM, Wellington CL, Wood JD, Herring WJ, Sawa A Hayden MR, Dawson VL, Dawson TM, Ross CA. Nuclear localization of a non-caspase truncation product of atrophin-1, with an expanded polyglutamine repeat, increases cellular toxicity. J Biol Chem 2003;278:13047-55.

13 MacDonald ME, Gusella JF. Huntington's disease: translating a CAG repeat into a pathogenic mechanism. Curr Opin Neurobiol 1996;6:638-43.

14 Housman D. Gain of glutamines, gain of function? Nat Genet 1995;10:3-4

15 Benson MD, Liepnieks J, Uemichi T, Wheeler G, Correa R. Hereditary renal amyloidosis associated with a mutant fibrinogen alpha-chain. Nat Genet 1993;3:252-5

16 Pepys MB, Hawkins PN, Booth DR, Vigushin DM, Tennent GA, Soutar AK, Totty N, Nguyen O, Blake CCF, Terry CJ, Feest TG, Zalin AM, Hsuan JJ. Human lysozyme gene mutations cause hereditary systemic amyloidosis. Nature 1993:362:553-7.

17 Driscoll M. Chalfie M. The mec-4 gene is a member of a family of Caenorhabditis elegans genes that can mutate to induce neuronal degeneration. Nature 1991;349:588-93. 